\title{
Novel Cysteine Tags for the Sequencing of Non-Tryptic Disulfide Peptides of Anurans: ESI-MS Study of Fragmentation Efficiency
}

\author{
Tatyana Y. Samgina, ${ }^{1}$ Egor A. Vorontsov, ${ }^{1}$ Vladimir A. Gorshkov, ${ }^{1}$ \\ Konstantin A. Artemenko, ${ }^{2}$ Ilya E. Nifant'ev, ${ }^{1}$ Basem Kanawati, ${ }^{3}$ \\ Philippe Schmitt-Kopplin, ${ }^{3}$ Roman A. Zubarev, ${ }^{4}$ Albert T. Lebedev ${ }^{1}$ \\ ${ }^{1}$ Department of Chemistry, Moscow State University, Russian Federation, 119991 Leninskie Gory 1/3, Moscow, Russia \\ ${ }^{2}$ Department of Physical and Analytical Chemistry, Uppsala University, Uppsala, Sweden \\ ${ }^{3}$ GSF Institute of Ecological Chemistry, Neuherberg, Germany \\ ${ }^{4}$ Department of Medicinal Biochemistry and Biophysics, Division of Molecular Biometry, Karolinska Institutet, Stockholm, \\ Sweden
}

\begin{abstract}
Mass spectrometry faces considerable difficulties in de novo sequencing of long non-tryptic peptides with S-S bonds. Long disulfide-containing peptides brevinins $1 \mathrm{E}$ and $2 \mathrm{Ec}$ from frog Rana ridibunda were reduced and alkylated with nine novel and three known derivatizing agents. Eight of the novel reagents are maleimide derivatives. Modified samples were subjected to MS/MS studies on FT-ICR and Orbitrap mass spectrometers using CAD/HCD or ECD/ETD techniques. Procedures, fragmentation patterns, and sequence coverage for two peptides modified with 12 tags are described. ECD/ETD and CAD fragmentation revealed complementary sequence information. Higher-energy collisionally activated dissociation (HCD) sufficiently enhanced $y$-ions formation for brevinin 1E, but not for brevinin 2Ec. Some novel tags [ $N$-benzylmaleimide, $N$-(2,6-dimethylphenyl)maleimide] along with known $\mathrm{N}$-phenylmaleimide and iodoacetic acid showed high total sequence coverage taking into account combined ETD and HCD fragmentation. Moreover, modification of long (34 residues) brevinin 2Ec with $N$-benzylmaleimide or $N$-(2,6-dimethylphenyl)maleimide yielded high sequence coverage and full C-terminal sequence determination with ECD alone.
\end{abstract}

Key words: Disulfide peptide, Brevinin, De novo sequencing, Cysteine tag, Maleimide, Electrospray ionization, ECD, ETD, CAD, HCD, FTICR-MS, Orbitrap

\section{Introduction}

$\mathrm{H}$ ost-defense antimicrobial peptides constitute the major portion of amphibian skin secretion being a part of their

Electronic supplementary material The online version of this article (doi:10.1007/s13361-011-0247-0) contains supplementary material, which is available to authorized users.

Correspondence to: Albert T. Lebedev; e-mail: a.lebedev@org.chem.msu.ru innate immunity. They may be treated as natural weapon against predators and pathogenic micro-organisms [1-12]. A search for new biologically active amphibian peptides followed by their sequencing represents a certain interest for the pharmaceutical industry [13].

Ranid frogs possess a wide variety of mature peptides, reflecting the adaptive abilities of these animals towards climatic and anthropogenic factors. The existing proteomic algorithm of the protein sequencing is not very efficient for the analysis of mixtures of natural peptides in general and 
amphibian skin secretions in particular. The principal issue involves the absence of genomic information, i.e., the absence of the sequence of the possible encoded peptides.

There are several other issues complicating the sequence of such peptides. Antimicrobial peptides (e.g., amphibian peptides) databases are still in the process of creation (APD: http://aps.unms.edu/AP/main.php; http://copewithcytokines. de). Ranid skin peptides (20-46 amino acid residues) usually exceed the optimal length for the efficient mass spectrometric sequencing. Routine approach dealing with trypsinolysis is also not very efficient as the high content of Lys and Arg residues results in formation of very short tryptic peptides, which are often lost during the analysis. Additional problem is connected with numerous peptides containing a cycle formed by intramolecular $\mathrm{S}-\mathrm{S}$ bond between two cysteine residues near their C-terminus. The feature is called "Rana box". This structural peculiarity complicates de novo sequencing of such peptides via mass spectrometry due to the lack of sequence ion coverage within the cycle. Thus, the problem of efficient de novo sequencing of long non-tryptic amphibian peptides cannot be considered resolved, despite the growing number of activation methods available in mass spectrometry.

Usually derivatization procedures used in mass spectrometry are aimed on improving sensitivity of analyses and on obtaining easily interpretable spectra with high sequence coverage. Comprehensive review of various reagents and modification procedures is presented in recent handbook [14]. Two main approaches are employed to facilitate mass spectrometric sequencing of disulfide-containing peptides: reduction followed by alkylation of free $-\mathrm{SH}$ groups or oxidation of $\mathrm{S}-\mathrm{S}$ bonds into sulfonic acids. Chemical modifications generally do have impact on peptide ionization and fragmentation processes. For example, complex procedure with recording $\mathrm{ECD}$ and $\mathrm{CAD}$ mass spectra (accurate measurements) of intact peptides followed by similar analysis of their oxidized and reduced-plus-alkylated modifications allows full sequence determination [15]. However it is a time consuming procedure requiring three injections and thorough processing of the recorded spectra.

Recently reduction/alkylation has been successfully implemented to "Rana box"-containing peptides of ranid frogs $[16,17]$. Frequently used reducing and alkylating agents (so-called cysteine tags) are summarized in recent review [18]. Dithiothreitol (DTT) is usually used for reduction, while iodoacetamide (IAA), iodoacetic acid (IAAC) and $\mathrm{N}$-substituted maleimides are common alkylating agents. MALDI-PSD, ESI-CAD, and ESI-ECD of derivatized peptides yield fragment ions often allowing determination of the whole C-terminal sequence $[19,20]$.

Oxidation of S-S bond with peroxyacids affords two sulfonic groups [21]. Oxidation is especially advantageous for MALDI-PSD sequencing because usually this method deals with singly protonated molecules. The forming sulfonic moieties at the $\mathrm{C}$-terminus improve fragment ion signals (especially $y$-ions), providing two additional mobile protons to the peptide backbone [22-26]. However, multiply protonated molecules of oxidized peptides were reported to be of weak abundance in ESI-MS/MS [27].

To analyze the influence of cysteine tags on the fragmentation of non-tryptic disulfide containing peptides of ranid frogs several new cysteine tags were synthesized (Figure 1):

All the tags effectively derivatize cysteine SH-groups of the reduced peptides [28]. The reaction involves both SHgroups while side reactions are not observed. MALDI PSD of two major peptides of the skin secretion of Rana ridibunda: brevinin 1E (2675 Da) and brevinin 2Ec (3516 Da) modified with all the tags mentioned in Figures 1 and 2 was reported in the recent paper [29].

The present paper deals with comparative study of ESIMS/MS fragmentation of the same natural model peptides (brevinin $1 \mathrm{E}$ and brevinin 2Ec) modified with common [iodoacetic acid (IAAC), iodoacetamide (IAA) and $\mathrm{N}$ phenylmaleimide (NPM)] and novel (Figure 1) cysteine tags. ECD, ETD, CAD, and HCD MS/MS spectra of these derivatized (12 tags) peptides were recorded with FT ICR and Orbitrap instruments. The influence of the tags structure on the sequence coverage and fragment ions abundance is described. The benefits and disadvantages of each of the mass spectrometric techniques used for the de novo sequencing of the long non-tryptic peptides are also discussed. The main task engaged the finding of a tag allowing de novo sequencing of such peptides in one injection.

\section{Experimental}

\section{Materials and Methods}

All solvents were of HPLC-grade (Acros, Geel, Belgium). Commercially available iodoacetic acid (IAAC) (Sigma, St. Louis, MO, USA) was recrystallized from hexane directly before the use. Commercially available iodoacetamide (IAA) and $N$-(1,1,1,2,2-pentafluoroprop-3-yl)-2-iodoacetamide (IAA-1) (Sigma, St. Louis, MO, USA) were used as received. Synthesis of $N$-phenylmaleimide (NPM) and the novel maleimide derivatives was described earlier [28].

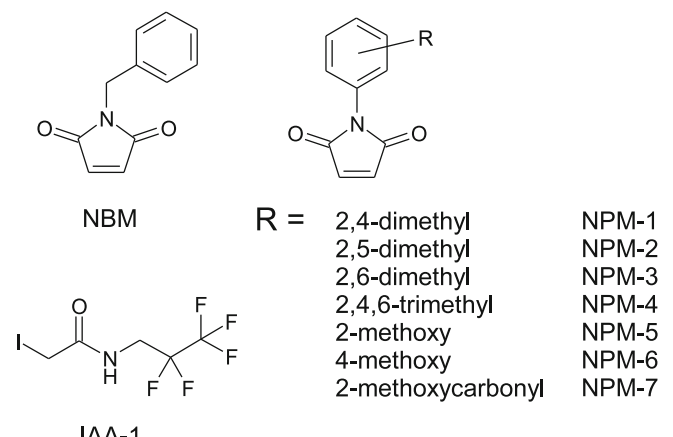

Figure 1. Novel cysteine tags used in present work 
(a)

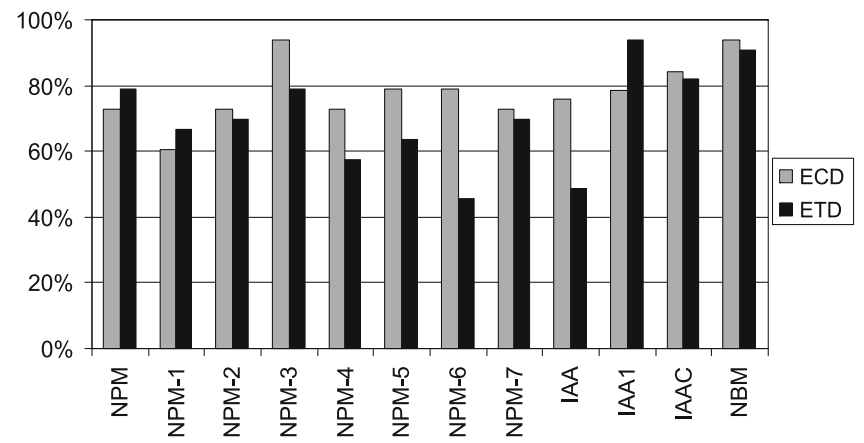

(c)

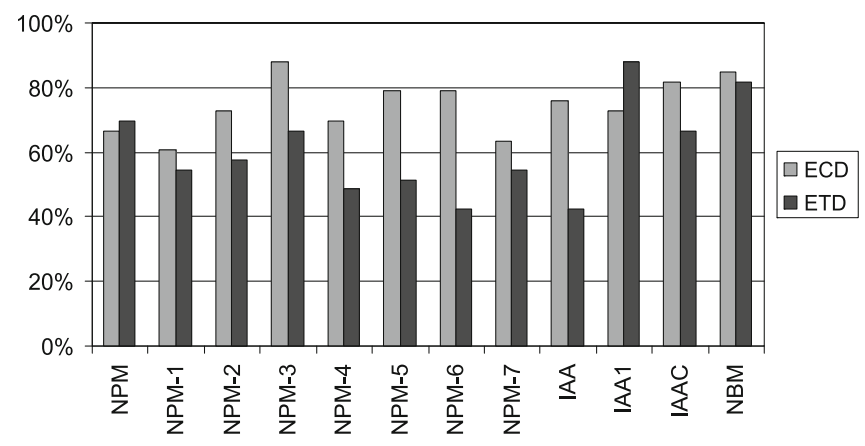

(b)

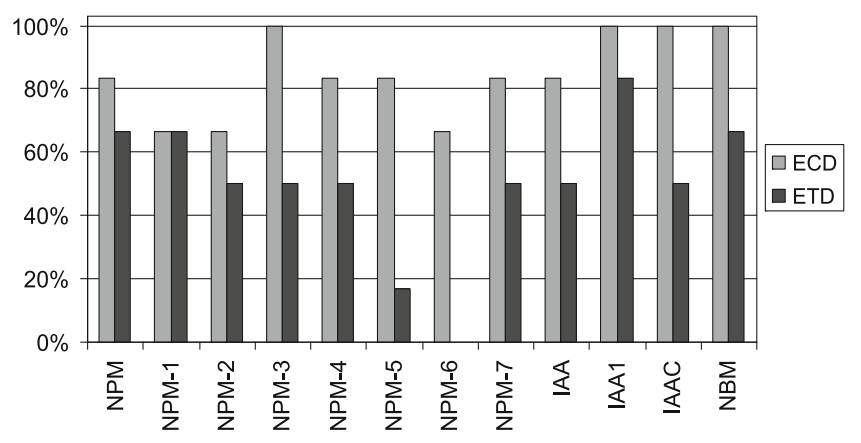

(d)

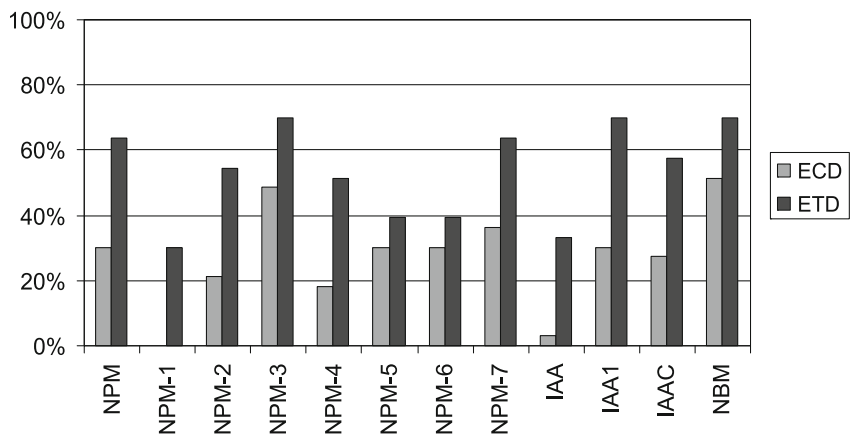

Figure 2. Sequence coverage for brevinin 2Ec with the studied tags in ECD (12-Tesla FT-ICR) or ETD (Orbitrap) modes: (a) overall coverage, (b) coverage in «Rana box», (c) $c$-ions coverage, (d) $z$-ions coverage

Novel tags employed in the present study are presented in Figure 1.

\section{Preparation of Skin Secretions}

Preparation of frog skin secretion was accomplished as described earlier [29, 30]. Both male and female Rana ridibunda species were caught in Moscow region. Adult animals were maintained in captivity at the Biology Department of the Moscow State University in conditions close to natural ones. Secretion from the skin glands was provoked by the mild electric stimulation during $40 \mathrm{~s}$ using laboratory electrostimulator ESL-1 (LP, USSR) equipped with platinum electrodes. The duration of impulse was $3 \mathrm{~ms}$ with amplitude of $10 \mathrm{~V}$ at $50 \mathrm{~Hz}$. The skin secretions were washed from the animals with MilliQ (Millipore, Billerica, MA, USA) water while an equal volume of methanol was immediately added to the aqueous solution. The mixtures were centrifuged and filtered through the PTF membrane filter $(0.45 \mu \mathrm{m})$. The solutions obtained were concentrated using rotary evaporator to a volume of $1 \mathrm{~mL}$ and lyophilized.

\section{Disulfide Bond Reduction and Alkylation}

Lyophilized crude secretion was redispersed in ammonium buffer (100 mM NH $\mathrm{NHCO}_{3}, \mathrm{pH}$ 8.0) with dithiothreitol at the concentration of $4 \mathrm{mM}$ [31]. The reaction solution was incubated for $1 \mathrm{~h}$ at $37^{\circ} \mathrm{C}$. Then, $10 \mathrm{mM}$ aqueous solution of iodoacetamide was added and the mixture was incubated for $1 \mathrm{~h}$ at $37^{\circ} \mathrm{C}$ in the dark. In the case of maleimides, alkylation was performed with $10 \mathrm{mM}$ tag solution in water/acetonitrile $(1: 1 \mathrm{vol} / \mathrm{vol})$ for $1 \mathrm{~h}$ at ambient temperature. Completion of reactions was checked by means of MALDI-MS.

\section{HPLC Separation of Reaction Mixtures}

Separation of the obtained reaction mixtures was performed with an HPLC system (Thermo Fisher Scientific Inc., San Jose, CA, USA) by injecting $50 \mu \mathrm{L}$ of a mixture into a reverse-phase chromatographic analytical column BiochemMack (BiochemMack, Moscow, Russia) $\mathrm{C}_{18}(5 \mu, 110 \AA, 4 \times$ $150 \mathrm{~mm}$ ) equilibrated with $10 \%$ acetonitrile/aqueous $0.1 \%$ trifluoroacetic acid. The peptides were purified using a linear gradient from $10 \%$ to $70 \%(40 \mathrm{~min})$ acetonitrile containing $0.1 \%$ trifluoroacetic acid. The flow rate was $0.8 \mathrm{~mL} / \mathrm{min}$. Two major peptides of Rana ridibunda skin secretion were employed in the studies: brevinin 1E: FLPLLAGLAANFLP KIFCKITRKC-OH (2675 Da, 24 residues); brevinin 2Ec: GILLDKLKNFAKTAGKGVLQSLLNTASCKLSGQC-OH (3516 Da, 34 residues). The first one possesses three basic amino acid residues (one Arg, two Lys) inside the cycle at the C-terminus due to $\mathrm{S}-\mathrm{S}$ bond formation between two Cys. Only one additional Lys is present at the backbone, being also close to the $\mathrm{C}$-terminus. The second peptide contains five Lys residues more or less randomly spread along the backbone, including one in the "Rana box." 


\section{Mass Spectrometry}

Mass spectra were obtained using electrospray ionization with several activation modes. Collisionally activated dissociation (CAD) experiments were performed with LTQ-FT Ultra (Thermo Fisher Scientific, Bremen, Germany) equipped with 7-Tesla magnet and nanoHPLC; detailed procedure described elsewhere [32]; LTQ Orbitrap Velos (Thermo Fisher Scientific, Bremen, Germany) with direct infusion (DI) of samples by offline nano-ESI source (Proxeon Biosystems, Odense, Denmark). Normalized collision energy was $35 \mathrm{u}$ for brevinin$1 \mathrm{E}$ and brevinin $2 \mathrm{Ec}(5+), 40 \mathrm{u}$ for brevinin $2 \mathrm{Ec}(4+)$, and $30 \mathrm{u}$ for brevinin 2Ec $(6+)$. Higher energy collision-induced dissociation (HCD) experiments were performed with Orbitrap instrument (DI). Normalized collision energy was $35 \mathrm{u}$ for brevinin $1 \mathrm{E}$ and brevinin $2 \mathrm{Ec}(6+$ and $5+)$ while for brevinin $2 \mathrm{Ec}(4+)$ it was $40 \mathrm{u}$. Electron capture dissociation (ECD) experiments were performed with LTQ-FT Ultra in the same manner as CAD experiments [32]; Apex Ultra Q-FTMS (Bruker Daltonics, Bremen, Germany) with 12-Tesla magnet in DI mode was used for brevinin $1 \mathrm{E} 4+$ state with electron energy $1 \mathrm{eV}$ and $100 \mathrm{~ms}$ pulse duration and for brevinin 2Ec 5+ state with electron energy $1 \mathrm{eV}$ and $75 \mathrm{~ms}$ duration. Orbitrap instrument was used for ETD experiments. Samples were infused directly into the ion source. Fluoranthene was used as reagent gas while $70 \mathrm{~ms}$ interaction time was chosen for brevinin $1 \mathrm{E}(4+)$ and brevinin $2 \mathrm{Ec}(4+)$ and $50 \mathrm{~ms}$ for brevinin $1 \mathrm{E}(5+)$ and brevinin-2Ec $(5+$ and $6+)$. In the conditions used (direct sample infusion), fragment ion intensities for all activation techniques were of the same order of magnitude in case of equal parent ions intensities.

In order to confirm that the disulfide bond reduction and alkylation reactions had proceeded to completion, the products were analyzed by MALDI-TOF mass spectrometer (Ultraflex II; Bruker Daltonics, Bremen, Germany). External calibration was made using a standard peptide mixture (PepMix-2; Bruker Daltonics, Bremen, Germany). The samples were deposited on the plate being mixed with saturated solution of 2,5-dihydroxybenzoic acid (DHB; Acros) in $0.1 \%$ TFA in acetonitrile/water (50:50 vol. \%).

\section{Results and Discussion}

Figure 2 represents ECD/ETD results for brevinin 2Ec derivatized with the studied tags (FTMS/Orbitrap).

Electron capture (12 Tesla) or electron transfer dissociation (Orbitrap) techniques showed rather promising results for the modified peptides studied. Sequence coverage for derivatized brevinin $2 \mathrm{Ec}$ was in the range from $\sim 45 \%$ to $\sim 94 \%$ (Figure $2 \mathrm{a}$ ). In most cases, ECD yields better or equal results compared to ETD. Traditionally used IAA and NPM tags gave less than $80 \%$ total sequence coverage for brevinin $2 \mathrm{Ec}$ in both ECD and ETD modes. 2,6-Dimethyl NPM (NPM-3) and NBM showed the best performance in ECD mode (see spectrum for NPM-3 modification in Figure 3), while pentafluoropropyl derivative

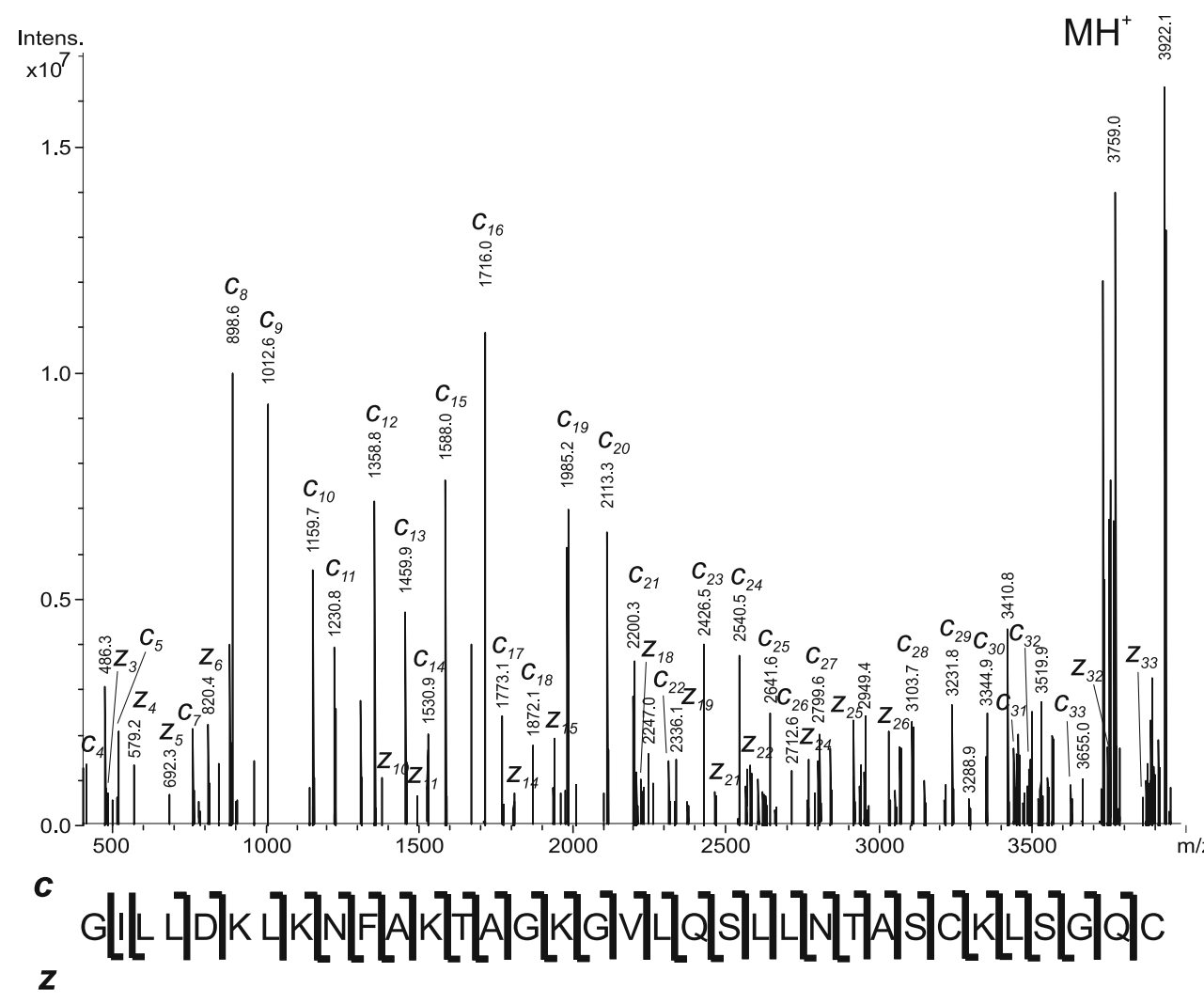

Figure 3. ECD spectrum of NPM-3-tagged brevinin 2Ec. Scheme of cleavages occurred is shown below the spectrum 
of iodoacetamide (IAA-1) demonstrated the same efficiency in ETD mode. In these three cases, $94 \%$ of coverage means that only two peptide bonds remained unbroken in the backbone. NBM tag worth special mentioning as it induced overall sequence coverage higher than $90 \%$ both for ECD and ETD modes.

C-terminal fragmentation of the modified ranid peptides is a matter of special concern. It gives a chance to identify the sequence inside the former "Rana box." This information often plays crucial role for classification of the peptide. The absence of Lys or Arg at C-terminus usually does not provide complete sequence information via MS/MS. Cterminal basic amino acid is absent in the structure of brevinin 2Ec "Rana box". Nevertheless, NBM, NPM-3, IAA-1, and IAAC allowed full sequence determination in "Rana box" with ECD (Figures $2 \mathrm{~b}$ and 3 ). The other tags furnished lower coverage. ECD performed better than ETD for "Rana box" sequencing irrespective of the tag used. Methoxy-groups in the aromatic ring (NPM-5, NPM-6) dramatically decrease the ETD fragmentation in the Cterminal part of brevinin 2Ec, with NPM-6 providing no fragmentation. Thus, a hypothesis may be put forward that the methoxy-substituted phenyl ring competes rather successfully for the electron transferring to the peptide molecule. As a result, this electron is not participating in the triggering of the backbone cleavage. IAA-1 tag may be mentioned as the best one as it demonstrated the best results

(a)

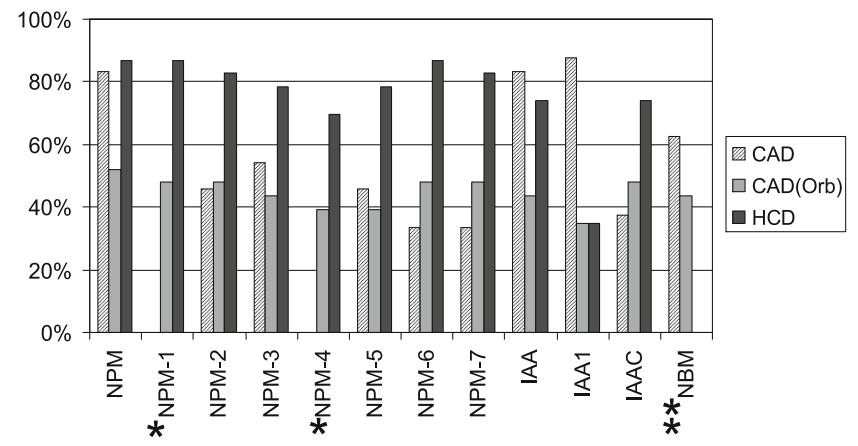

(c)

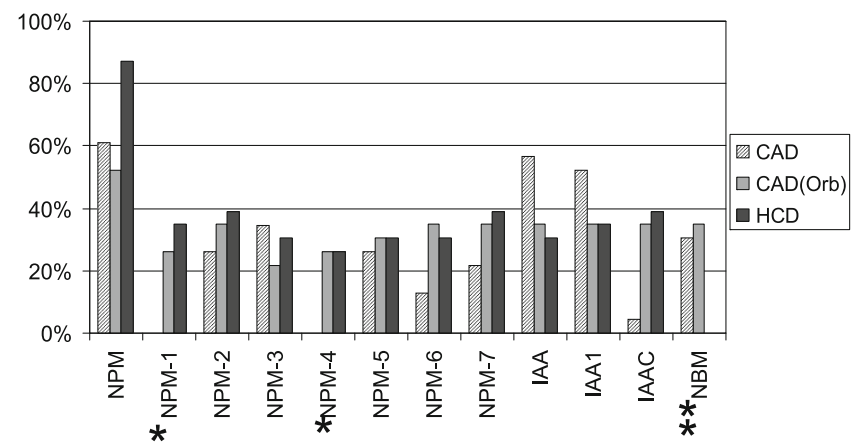

in ETD and ECD modes. NBM tag performance may be emphasized as well.

Contribution of $c$ - and $z$-ions in the overall sequence coverage is represented in Figure $2 \mathrm{c}$ and d. ECD outperforms ETD in terms of $c$-ions formation for all the tags except NPM and IAA-1. In contrast, ETD $z$-series are considerably longer than ECD ones for all the tags. Thus, for 2,4-dimethyl derivative (NPM-1) ECD yields no $z$-ions (ETD spectrum contains both ion series). Theoretically, an efficient tag should provide high coverage for both ion series as the reliability of the sequencing is higher in the presence of the complementary ion pairs. In case of ECD/ETD fragmentation of brevinin 2Ec such tags are NMP-3, IAA1, IAAC, NBM. An excellent performance of NBM demonstrates that electronic effects of the tag are not so relevant for increasing the sequence coverage. Since the aromatic ring of NBM is separated from maleimide moiety by $\mathrm{CH}_{2}$-group, conjugation with the rest of the molecule is impossible. As a result, mesomeric effects are impossible while inductive effects are notably suppressed. On the other hand, a specially synthesized $N$-methylmaleimide showed poor results even in MALDI [29]. Thus, the benzene ring of the tag somehow plays a role in promoting fragmentation of the backbone under the conditions studied.

In general, collisionally activated dissociation methods (CAD, HCD) yield worse overall coverage for brevinin 2Ec than ECD/ETD do (Figure S1 in Electronic Supplementary (b)

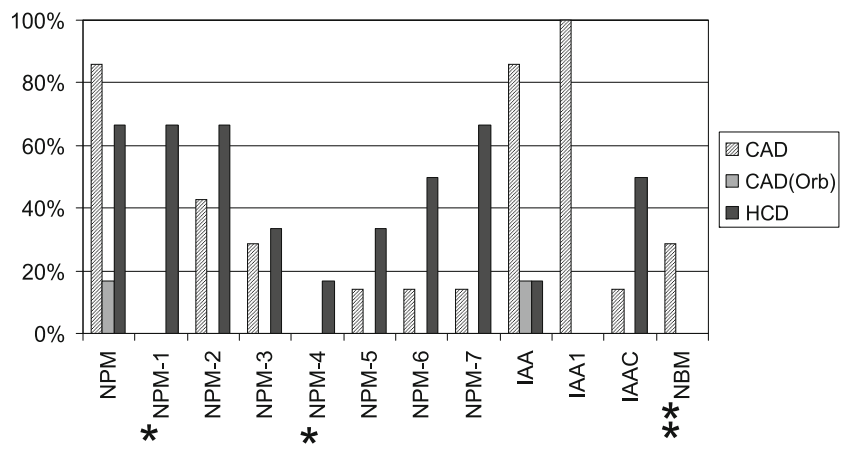

(d)

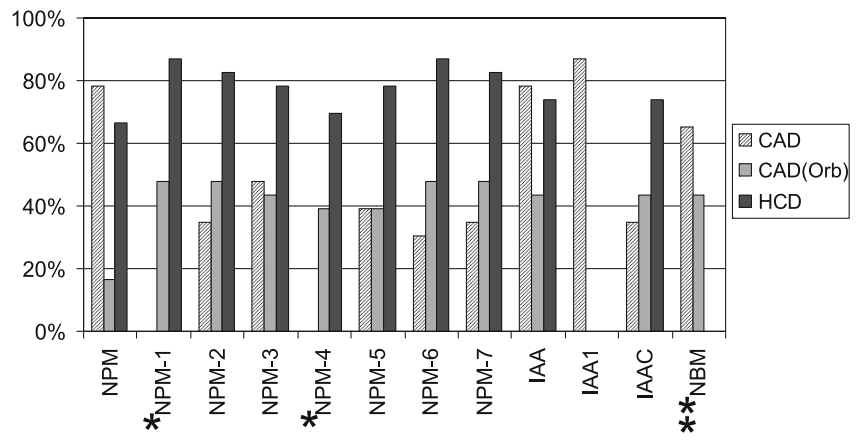

Figure 4. Sequence coverage for brevinin $1 \mathrm{E}$ with the studied tags in CAD (7-Tesla FT-ICR), CAD and HCD (Orbitrap) modes: (a) overall coverage, (b) coverage in "Rana box", (c) $b$-ions coverage, (d) y-ions coverage. *CAD (7-Tesla FT-ICR) not measured for these tagged peptides. ${ }^{\star} \mathrm{HCD}$ not measured for this tagged peptide 
Material for this article). Only NPM and its 2,6-dimethyl derivative (NPM-3) provided $80 \%$ overall sequence coverage (Figure S1A). Modifications with other tags give only $20 \%-70 \%$ coverage. It is hardly possible to select preferential type of experimental conditions. The results were fairly similar for all three experiments, independent of instrument or CAD versus HCD.

In most cases the "Rana box" sequence of the tagged peptide was fully determined by means of CAD (Figure S1B). Modification with NBM, NPM, and NPMs$3,-5,-7$ resulted in full "Rana box" sequence coverage with all three CAD variations studied. Therefore, $\mathrm{CAD} / \mathrm{HCD}$ appeared to be more efficient for the determination of Cterminal sequence of 34 residues long brevinin $2 \mathrm{Ec}$ than ECD/ETD approach. However NBM, NPM-3, IAA-1, and IAAC may be considered as the most promising tags allowing full sequence determination in "Rana box" with ECD and CAD.

Considering $b$ - and $y$-ion series separately (Figure S1C and $\mathrm{D}$, respectively) it should be mentioned that for all the tags $b$-series prevail over $y$-series, much more significantly than $c$-series over $z$-series in ECD/ETD spectra. This fact

Table 1. Results for Derivatized Brevinins 1E and 2Ec Sequence Coverage. The Best Fragmentation Method for each Peptide-tag Combination Outlined in the Rightmost Column, the Best Coverage and Method for each Peptide Marked Grey

\begin{tabular}{|c|c|c|c|c|c|c|c|}
\hline \multicolumn{8}{|c|}{ Brevinin 1E } \\
\hline & \multicolumn{6}{|c|}{ Coverage, \% } & \multirow[b]{2}{*}{ Best } \\
\hline & ECD 7T & ECD 12T & ETD & CAD 7T & CAD Orb & HCD & \\
\hline NPM & 45.8 & 87.0 & 39.1 & 83.3 & 52.2 & 87.0 & ECD 12T \\
\hline NPM-1 & n.d. & 52.2 & 91.3 & n.d. & 47.8 & 87.0 & ETD \\
\hline NPM-2 & 66.7 & 52.2 & 78.3 & 45.8 & 47.8 & 82.6 & $\mathrm{HCD}$ \\
\hline NPM-3 & 75.0 & 73.9 & 82.6 & 54.2 & 43.5 & 78.3 & ETD \\
\hline NPM-4 & n.d. & 43.5 & 34.8 & n.d. & 39.1 & 69.6 & $\mathrm{HCD}$ \\
\hline NPM-5 & n.d. & 82.6 & 78.3 & 45.8 & 39.1 & 78.3 & ECD 12T \\
\hline NPM-6 & 73.9 & 82.6 & n.d. & 33.3 & 47.8 & 87.0 & $\mathrm{HCD}$ \\
\hline NPM-7 & n.d. & 69.6 & 65.2 & 33.3 & 47.8 & 82.6 & $\mathrm{HCD}$ \\
\hline NBM & 70.8 & 26.1 & 69.6 & 62.5 & 43.5 & n.d. & ECD 7T \\
\hline IAA & 83.3 & 87.0 & 69.6 & 83.3 & 43.5 & 73.9 & ECD 12T \\
\hline IAA1 & 83.3 & 87.0 & 39.1 & 87.5 & 34.8 & 34.8 & ECD 12T \\
\hline IAAC & 70.8 & 82.6 & 78.3 & 37.5 & 47.8 & 73.9 & ECD 12T \\
\hline \multicolumn{8}{|c|}{ Brevinin 2Ec } \\
\hline & \multicolumn{6}{|c|}{ Coverage, \% } & \\
\hline & ECD 7T & ECD 12T & ETD & CAD 7T & CAD Orb & $\mathrm{HCD}$ & Best \\
\hline NPM & 91.2 & 72.7 & 78.8 & 79.4 & 57.6 & 57.6 & ECD 7T \\
\hline NPM-1 & n.d. & 60.6 & 66.7 & 20.6 & 51.5 & 57.6 & ETD \\
\hline NPM-2 & n.d. & 72.7 & 69.7 & 38.2 & 63.6 & 69.7 & ECD 12T \\
\hline NPM-3 & 79.4 & 93.9 & 78.8 & 79.4 & 48.5 & 51.5 & ECD 12T \\
\hline NPM-4 & n.d. & 72.7 & 57.6 & n.d. & 48.5 & 54.5 & ECD 12T \\
\hline NPM-5 & n.d. & 78.8 & 63.6 & 58.8 & 54.5 & 66.7 & ECD 12T \\
\hline NPM-6 & n.d. & 78.8 & 45.5 & 38.2 & 60.6 & 66.7 & ECD 12T \\
\hline NPM-7 & n.d. & 72.7 & 69.7 & 55.9 & 57.6 & 54.5 & ECD 12T \\
\hline NBM & 91.2 & 93.9 & 90.9 & 61.8 & 57.6 & 63.6 & ECD 12T \\
\hline IAA & 85.3 & 75.8 & 48.5 & 64.7 & 54.5 & 57.6 & ECD 7T \\
\hline IAA1 & n.d. & 78.7 & 93.9 & 29.4 & 60.6 & 33.3 & ETD \\
\hline IAAC & n.d. & 84.4 & 81.8 & 37.5 & 66.7 & 36.4 & ECD 12T \\
\hline
\end{tabular}

n.d. $=$ not determined 
resembles the structural peculiarity of brevinin 2Ec having 5 lysine residues spread along the backbone.

Brevinin $1 \mathrm{E}$, being the second major peptide in the skin secretion of Rana ridibunda, contains arginine residue in its "Rana box" region. Although brevinin $1 \mathrm{E}$ is considerably shorter (24 versus 34 amino acids) than brevinin $2 \mathrm{Ec}$, it can be difficult to obtain the full sequence of this peptide since all four basic amino acids are situated close to C-terminus. Tagged peptide dissociates rather intensively, showing total sequence coverage in ECD mode up to $87 \%$ for NPM, IAA, IAA-1 tags with FT-ICR 12-Tesla instrument (Figure S2 in Electronic Supplementary Material). ETD coverage (Orbitrap) reached $91 \%$ for NPM-1 tag (Figure S2A). NPM-3 and NBM, which were the most effective tags for brevinin 2Ec, show slightly poorer performance: $83 \%$ (NPM-3, ETD) and $71 \%$ (NBM, ECD). NPM-1, NPM-2, and NPM-3 tags are the positional isomers with two methyl groups in the aromatic ring. They performed nicely in ETD experiments. However, introduction of the third methyl group into the aromatic ring (NPM-4) dramatically reduced the sequence coverage (Figure S2A). Since the differences in electrondonating properties of these compounds are rather small the observed decrease may be due to steric effects.

The "Rana box" sequence ion coverage was also high: full sequence determination in this C-terminal region is obtainable with either ECD or ETD for all the tags excluding NPM-4 and NPM-7 (Figure S2B). High "Rana box" coverage may be a consequence of the presence of two lysine and one arginine residues near C-terminus. Thus, ETD allows full sequence coverage inside "Rana box" for brevinin $1 \mathrm{E}$ with the majority of the tags (NPM-1, NPM-2, NPM-3, NPM-5, IAA, IAAC, and NBM), while for brevinin 2Ec lacking these basic residues its performance was notably worse (Figure 2b).

There are not any notable predominance of $z$ - or $c$-ions (Figure S2C and D). The abundance of these ion series is highly dependent on the tag structure and fragmentation activation method. Thus 3.5 times more $z$-ions are formed during fragmentation of brevinin $1 \mathrm{E}$ tagged with methoxylated NPM-6 (ECD, 7-Tesla). In contrast, dimethylated NPM-2 provides two times more $c$-ions. Iodoacetamides IAA and IAA-1 result in similar quantities of $c$ - and $z$-ions. Similar results were obtained for the fragmentation of brevinin $1 \mathrm{E}$ with various tags in ECD (12-Tesla) and ETD modes.

Figure 4 represents the results of $\mathrm{CAD} / \mathrm{HCD}$ fragmentation of tagged brevinin 1E. There is a noticeable difference between $\mathrm{CAD}$ and HCD fragmentation for brevinin 1E. NPM, IAA, and IAA-1 show high overall sequence coverage (over $80 \%$ ) in CAD (LTQ-FT), while modifications with other tags yield less than $60 \%$ coverage (Figure $4 a$ ). Orbitrap in CAD mode provides relatively poor spectra with less than $50 \%$ coverage. However, fragmentation is dramatically improving with the use of HCD revealing up to $85 \%$ of the sequence (NPM, NPM-1, NPM-6).

The sequence ion coverage inside the "Rana box" is rather variable from one tag to another. Full "Rana box" sequence was determined only for IAA-1 in CAD (LTQ-FT) conditions (Figure 4b). HCD also could not provide better results for this piece of the peptide.

\section{Brevinin 1E}
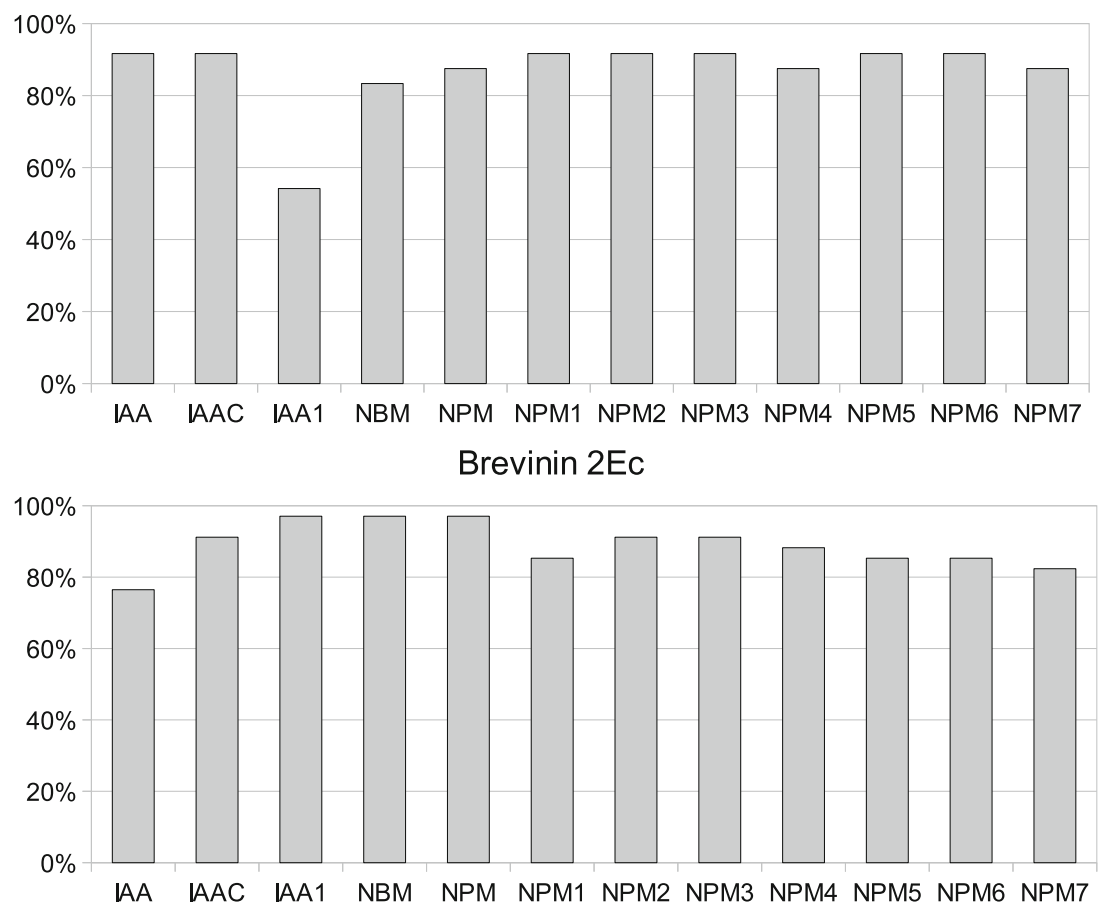

Figure 5. Total sequence coverage obtained in a single injection using alternating recording of ETD/HCD fragmentation of derivatized peptides 
$\mathrm{HCD}$ is similar to $\mathrm{CAD}$ in production of $b$-ions (Figure $4 \mathrm{c}$ ), but it sufficiently extends $y$-series (Figure $4 \mathrm{~d}$ ), leading to high total coverage. This effect was not observed for brevinin $2 \mathrm{Ec}$. It is also worth mentioning the poor performance of IAA-1 tag in Orbitrap experiments, although this iodoacetamide derivative with an electron withdrawing substituent was the best one in
CAD on FT-ICR 7-Tesla instrument providing $90 \%$ total sequence and being the single tag showing $100 \%$ sequence in "Rana box" and the longest $y$-series.

Table 1 demonstrates the best results for each of 12 studied tags for brevinins $1 \mathrm{E}$ and $2 \mathrm{Ec}$ sequence. The best results for brevinin $2 \mathrm{Ec}$ sequencing were obtained in $\mathrm{ECD}$

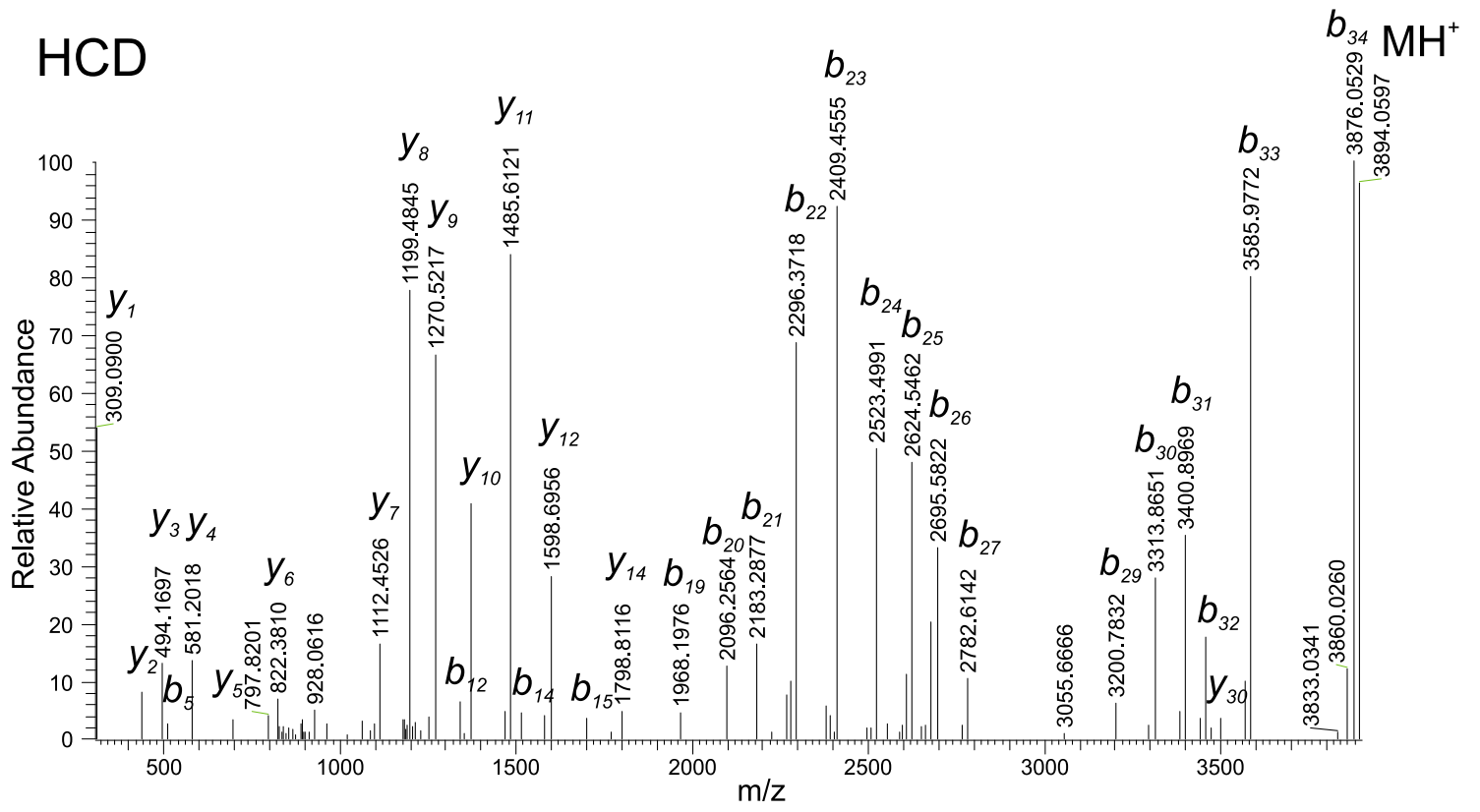

\section{ETD}
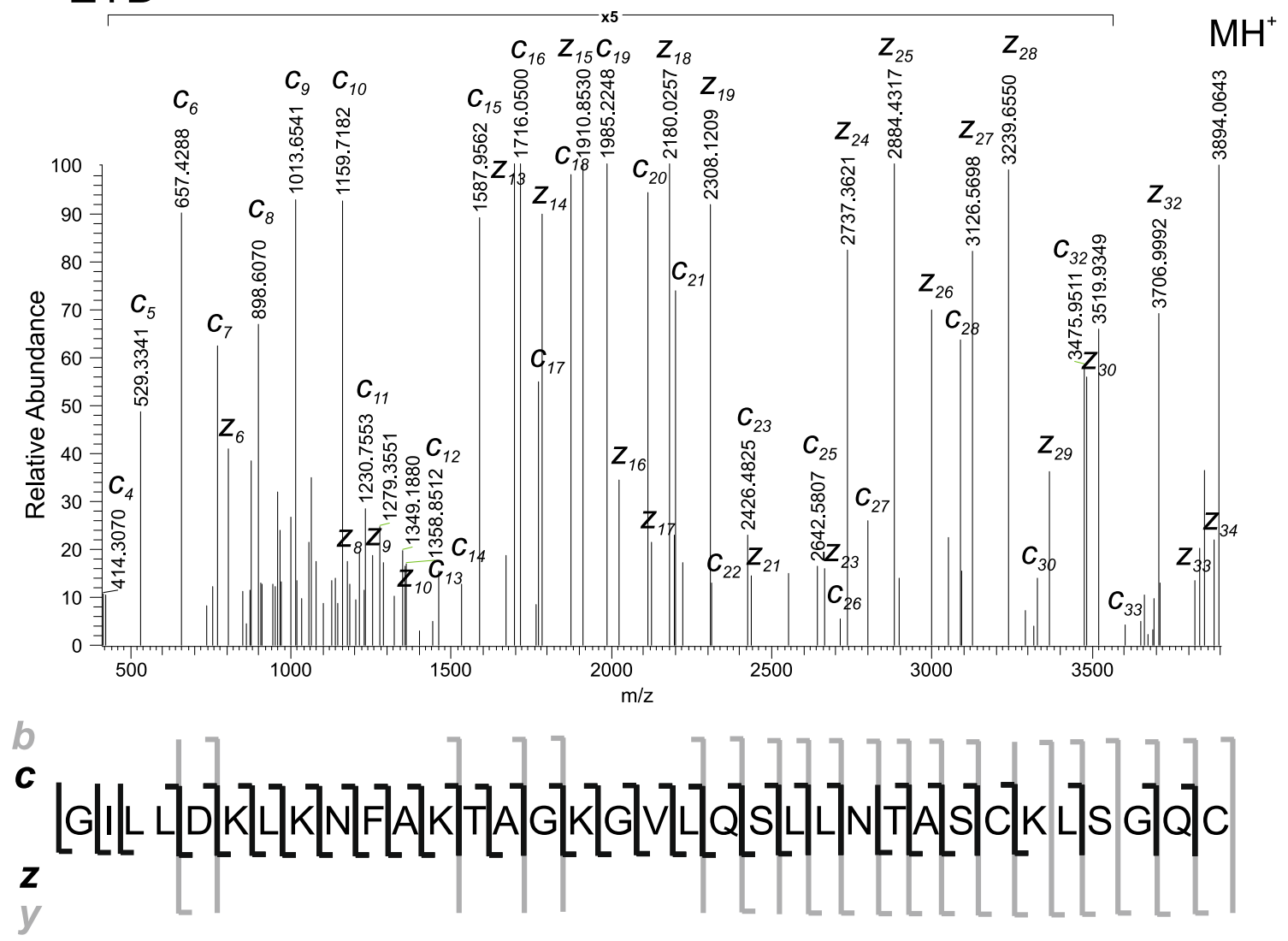

Figure 6. HCD and ETD spectra of NBM-tagged brevinin 2Ec. Scheme of cleavages occurred is shown below the spectra 
mode with 12T FT-MS instrument for NBM and NPM-3 tags and in ETD mode for IAA-1 tag. In these cases, only two peptide bonds out of 34 remain unbroken.

The best result for brevinin $1 \mathrm{E}$ sequencing was obtained in ETD mode for NPM-1 tag (91\%). Slightly poorer results were obtained in ECD mode on FT-ICR 12-Tesla instrument for IAA, IAA-1, NPM tags ( $87 \%$ each) and in HCD mode (Orbitrap) for NPM-6 tag (87\%).

Since the instruments used in this study are capable of switching between recording $\mathrm{CAD} / \mathrm{ECD}$ or $\mathrm{CAD} / \mathrm{ETD}$ spectra, it was important to understand what level of the sequence coverage can be achieved with the use of a specific tag in one injection. Figure 5 represents these values for both model brevinins. The results clearly demonstrate quite high sequence coverage for both peptides for the majority of the tags used. IAAC, NPM-2, and NPM-3 demonstrated higher than $90 \%$ coverage obtained in one injection. Thus it is worth testing these tags with other natural peptides with C-terminal cycle. The most widely used for the alkylation of the reduced thiol groups iodoacetamide showed almost $20 \%$ lower coverage than the most efficient tags used in the present study. $N$-benzylmaleimide mentioned already above should be also taken into account.

Figure 6 shows the HCD and ETD mass spectra of brevinin 2Ec modified with this tag. The detected peptide bond cleavages are marked below the spectra. As one can see, two complementary spectra of brevinin 2Ec (34 residues long) contain fragment ions proving all the peptide bond cleavages excluding one: $\mathrm{Leu}^{3}-\mathrm{Leu}^{4}$. Only two cleavages are defined with one ion: $z_{32}$ and $z_{33}$, while the predominant portion of sequence is confirmed by a pair of complementary ions in one or two spectra. This fact significantly increases the reliability of the sequencing. Thus, we recommend this tag for the de novo sequencing of long non-tryptic peptides.

\section{Conclusions}

Twelve tags belonging to maleimide and iodoacetamide groups were tested in order to find the most efficient ones for the de novo sequencing of long non-tryptic peptides with $\mathrm{S}-\mathrm{S}$ bond at the C-terminus. A number of the tags demonstrated excellent efficiency allowing complete sequence coverage of the "Rana box" region, which usually remains hidden in the spectra of intact peptides. Although none of the tags demonstrated $100 \%$ overall sequence coverage, several of them are worth special mention as they provided more than $90 \%$ sequence coverage for both tested peptides in one injection. These tags are: iodoacetic acid (IAAC), 2,5-dimethylphenylmaleimide (NPM2), 2,6-dimethylphenylmaleimide (NPM-3), and benzylmaleimide (NBM). Their efficiency will be tested on the broader range of non-tryptic peptides with $\mathrm{S}-\mathrm{S}$ bonds in the molecule.

\section{References}

1. Bevins, C.L., Zasloff, M.: Peptides from Frog Skin. Annu. Rev. Biochem. 59, 395-414 (1990)
2. Clarke, B.T.: The Natural History of Amphibian Skin Secretions, Their Normal Functioning, and Potential Medical Applications. Biol. Rev. Camb. Philos. Soc. 72(3), 365-379 (1997)

3. Severini, C., Improta, G., Tachykinins, A., et al.: In Handbook of Biologically Active Peptides, pp. 261-268. Academic Press, New York (2006)

4. Erspamer, V., Falconieri Erspamer, G., Cei, J.M.: Active Peptides in the Skins of 230 American Amphibian Species. Comp. Biochem. Physiol. C 85(1), 125-137 (1986)

5. Roseghini, M., Falconieri Erspamer, G., Severeni, C., Simmaco, M.: Biogenic Amines and Active Peptides in Extracts of the Skin of 32 European Amphibian Species. Comp. Biochem. Physiol. C 94(2), 455460 (1989)

6. Pukala, T.L., Bowie, J.H., Maselli, V.M., Musgrave, I.F., Tyler, M.J.: Host-Defense Peptides from the Glandular Secretions of Amphibians: Structure and Activity. Nat. Prod. Rep. 23(3), 368-393 (2006)

7. Simmaco, M., Mignogna, G., Barra, D.: Antimicrobial Peptides from Amphibian Skin: What Do They Tell Us? Biopolymers 47(6), 435-450 (1998)

8. Conlon, J.M., Kolodziejek, J., Nowotny, N.: Antimicrobial Peptides from Ranid Frogs: Taxonomic and Phylogenetic Markers and a Potential Source of New Therapeutic Agents. Biochim. Biophys. Acta 1696(1), 1-14 (2004)

9. Doyle, J., Brinkworth, C.S., Wegener, K.L., Carver, J.A., Llewellyn, L.E., Olver, I.N., Bowie, J.H., Wabnitz, P.A., Tyler, M.J.: nNOS Inhibition, Antimicrobial, and Anticancer Activity of the Amphibian Skin Peptide, Citropin 1.1 and Synthetic Modifications. The Solution Structure of a Modified Citropin 1.1. Eur. J. Biochem. 270(6), 1141-1153 (2003)

10. Li, J., Xu, X., Xu, C., Zhou, W., Zhang, K., Yu, H., Zhang, Y., Zheng, Y., Rees, H.H., Lai, R., Yang, D., Wu, J.: Anti-Infection Peptidomics of Amphibian Skin. Mol. Cell. Proteom. 6(5), 882-894 (2007)

11. Mangoni, M.L., Rinaldi, A.C., Di Giulio, A., Mignogna, G., Bozzi, A., Barra, D., Simmaco, M.: Structure-Function Relationships of Temporins, Small Antimicrobial Peptides from Amphibian Skin. Eur. J. Biochem. 267(5), 1447-1454 (2000)

12. Conlon, J.M.: The Therapeutic Potential of Antimicrobial Peptides from Frog Skin. Rev. Med. Microbiol. 15(1), 17-25 (2004)

13. Peptide Antibiotics-Discovery, Modes of Action, and Applications, Dutton C. D.; Haxell M. A.; McArthur H. A. I.; Wax R. G., Eds.; Pfizer Global Research: Groton, CT, 2001; pp 308.

14. Zaikin, V., Halket, J.: Soft Ionization Mass Spectrometry of Large Molecules: A Handbook of Derivatives for Mass Spectrometry, p. 299. IM Publications LLP, Charlton, UK (2009)

15. Gorshkov, V.A., Samgina, T.Y., Vorontsov, E.A., Artemenko, K.A., Zubarev, R.A.: Mass Spectrometry to Establish the Skin Secretory Peptidome of Ranid and Hylid Frogs, p. 52. Proceedings of the 1st Middle Eastern and Mediterranean Sea Region Countries Mass Spectrometry Workshop, Rehovot, Israel (2010)

16. Samgina, T.Y., Artemenko, K.A., Gorshkov, V.A., Ogourtsov, S.V., Zubarev, R.A., Lebedev, A.T.: De Novo Sequencing of Peptides Secreted by the Skin Glands of the Caucasian Green Frog Rana ridibunda. Rapid Commun. Mass Spectrom. 22, 3517-3525 (2008)

17. Samgina, T.Y., Artemenko, K.A., Gorshkov, V.A., Ogourtsov, S.V., Zubarev, R.A., Lebedev, A.T.: Mass Spectrometric Study of Peptides Secreted by the Skin Glands of the Brown Frog Rana arvalis from the Moscow Region. Rapid Commun. Mass Spectrom. 23, 1241-1248 (2009)

18. Artemenko, K.A., Samgina, T.Y., Lebedev, A.T.: Peptide De Novo Sequencing by Mass Spectrometry. Mass-Spektrometria 3(4), 225-254 (2006)

19. Samgina, T.Y., Artemenko, K.A., Gorshkov, V.A., Lebedev, A.T., Nielsen, M.L., Savitski, M.L., Zubarev, R.A.: Electrospray Ionization Tandem Mass Spectrometry Sequencing of Novel Skin Peptides from Ranid Frogs Containing Disulfide Bridges. Eur. J. Mass Spectrom. 13 (2), 155-163 (2007)

20. Samgina, T.Y., Artemenko, K.A., Gorshkov, V.A., Poljakov, N.B., Lebedev, A.T.: Oxidation Versus Carboxyamidomethylation of S-S Bond in Ranid Frog Peptides: Pro and Contra for De Novo MALDI-MS Sequencing. J. Am. Soc. Mass Spectrom. 19(4), 479487 (2008)

21. Hirs, C.H.W., Hirs, C.H.W.: Performic Acid Oxidation: Methods in Enzymology, p. 197. Academic Press, Oxford, Great Britain (1967)

22. Bartlet, O., Yang, C.Y., Gaskell, S.J.: Influence of Cysteine to Cysteic Acid Oxidation on the Collision-Activated Decomposition of Proto- 
nated Peptides-Evidence for Intraionic Interactions. J. Am. Soc. Mass Spectrom. 3(4), 337-344 (1992)

23. Dai, J., Zhang, Y., Wang, J., Li, X., Lu, Z., Cai, Y., Qian, X.: Identification of Degradation Products Formed During Performic Oxidation of Peptides and Proteins by High-Performance Liquid Chromatography with Matrix-Assisted Laser Desorption/Ionization and Tandem Mass Spectrometry. Rapid Commun. Mass Spectrom. 9 (9), 1130-1138 (2005)

24. Keough, T., Youngquist, R.S., Lacey, M.P.: Sulfonic Acid Derivatives for Peptide Sequencing by MALDI MS. Anal. Chem. 75(7), 157-165 (2003)

25. Wang, D., Kalb, S.R., Cotter, R.J.: Improved Procedures for NTerminal Sulfonation of Peptides for Matrix-Assisted Laser Desorption/Ionization Post-Source Decay Peptide Aequencing. Rapid Commun. Mass Spectrom. 18(1), 96-102 (2004)

26. Hellmann, U., Bhikhabhai, R.: Easy Amino Acid Sequencing of Sulfonated Peptides Using Post-Source Decay on a Matrix-Assisted Laser Desorption/Ionization Time-of-Flight Mass Spectrometer Equipped with a Variable Voltage Reflector. Rapid Commun. Mass Spectrom. 16(19), 1851-1859 (2002)

27. Dai, J., Wang, J., Zhang, Y., Lu, Z., Yang, B., Li, X., Lu, Z., Cai, Y., Qian, X.: Enrichment and Identification of Cysteine-Containing Peptides from Tryptic Digests of Performic Oxidized Proteins by Strong Cation Exchange LC and MALDI-TOF/TOF MS. Anal. Chem. 77(23), 7594-7604 (2005)
28. Samgina, T.Y., Gorshkov, V.A., Vorontsov, E.A., Bagrov, V.V., Nifant'ev, I.E., Lebedev, A.T.: New Cysteine-Modifying Reagents: Efficiency of Derivatization and Influence on the Signals of the Protonated Molecules of Disulfide-Containing Peptides in Matrix-Assisted Laser Desorption/Ionization Mass Spectrometry. J. Anal. Chem. 65(13), 1320-1327 (2010). Translated from: Samgina, T. Y.; Gorshkov, V. A.; Vorontsov, E. A.; Bagrov, V. V.; Nifant'ev, I. E.; Lebedev, A. T. New Cysteine-Modifying Reagents: Efficiency of Derivatization and Influence on Yield of Signals of Protonated Disulfide-Containing Peptide Molecules in Matrix-Assisted Laser Desorption/Ionization Mass Spectrometry. Mass Spektrometria 6(3), 178-186 (2009)

29. Vorontsov, E.A., Samgina, T.Y., Gorshkov, V.A., Poljakov, N.B., Nifant'ev, I.E., Lebedev, A.T.: MALDI-PSD Fragmentation of the Cysteine-Containing Amphibian Peptides with Novel Cysteine tags. Eur. J. Mass Spectrom. 17(1), 73-83 (2011)

30. Tyler, M.J., Stone, D.J., Bowie, J.H.: A Novel Method for the Release and Collection of Dermal. Glandular Secretions from the Skin of Frogs. J. Pharmacol. Toxicol. Methods 28(4), 199-200 (1992)

31. Allen, G.: In: Work, T.S., Burdon, R.H. (eds.) Sequencing of Proteins and Peptides, Laboratory Techniques in Biochemistry and Molecular Biology, p. 327. Elsevier, North Holland, Amsterdam (1981)

32. Kocher, T., Savitski, M.M., Nielsen, M.L., Zubarev, R.A.: PhosTShunter: A Fast and Reliable Tool to Detect Phosphorylated Peptides in Liquid Chromatography Fourier Transform Tandem Mass Spectrometry Data Sets. J. Proteome Res. 5(3), 659-668 (2006) 